\title{
Determinação de Mn e Zn por FAAS após tratamento em microescala de óxidos mistos para sua caracterização
}

\section{Determination of Mn e Zn by FAAS after treatment in micro-scale of the mixed oxides for their characterization}

\author{
Camila Corrêa Pereira ${ }^{1}$; Eliézer Quadro Oreste ${ }^{2}$; Alexander Ossanes de Souza ${ }^{3}$; Marcela \\ Betta Olimpio do Nascimento ${ }^{4}$; Rodrigo José Corrêa ${ }^{5}$; Daniela Bianchini ${ }^{6}$; Anderson \\ Schwingel Ribeiro ${ }^{7}$; Emerson Schwingel Ribeiro ${ }^{8}$
}

\begin{abstract}
Resumo
Este trabalho apresenta uma metodologia na determinação de Mn e Zn em amostras a base de sílica por espectrometria de absorção atômica com chama após tratamento em microescala para a caracterização de óxidos mistos. Para o tratamento das amostras, foram utilizados cerca de $10 \mathrm{mg}$ de óxido misto, 50 $\mu \mathrm{L}$ de $\mathrm{HF}, 50 \mu \mathrm{L}$ de $\mathrm{HNO}_{3}, 25 \mu \mathrm{L}$ de $\mathrm{H}_{2} \mathrm{O}_{2}$ com aquecimento por micro-ondas e diluição final de $2,0 \mathrm{~mL}$. $\mathrm{O}$ procedimento foi aplicado para a determinação de $\mathrm{Mn}$ e $\mathrm{Zn}$, e os resultados foram comparados com a técnica semiquantitativa de fluorescência de raios-X. A exatidão foi avaliada por testes de recuperação (93,5 a 100,7\%) e RSD abaixo de $9 \%$.
\end{abstract}

Palavras-chave: Óxidos mistos. Preparação de Amostras em Microescala. FAAS.

\begin{abstract}
This paper presents a methodology for the determination of $\mathrm{Mn}$ and $\mathrm{Zn}$ in samples based on silica by flame atomic absorption spectrometry after treatment in micro-scale for their characterization of mixed oxides. For treatment of samples, about $10 \mathrm{mg}$ of mixed oxide, $50 \mu \mathrm{L}$ of $\mathrm{HF}, 50 \mu \mathrm{L}$ of $\mathrm{HNO}_{3}, 25 \mu \mathrm{L}$ of $\mathrm{H}_{2} \mathrm{O}_{2}$ with microwave heating and final dilution to $2.0 \mathrm{~mL}$ were employed. The procedure was applied for the determination of $\mathrm{Mn}$ and $\mathrm{Zn}$, and the results were compared with the semiquantitative technique of X-ray fluorescence. The accuracy was evaluated by recovery tests (93.5 to 100.7\%) and RSD below 9\%.
\end{abstract}

Keywords: Mixed oxides. Sample preparation in micro-scale. FAAS

\footnotetext{
${ }^{1}$ Aluna de Doutorado - Universidade Federal de Pelotas (UFPel), Laboratório de Metrologia Química, Programa de Pós-Graduação em Química, 96160-000 Capão do Leão-RS, Brasil.

${ }^{2}$ Aluno de Doutorado - Universidade Federal de Pelotas (UFPel), Laboratório de Metrologia Química, Programa de Pós-Graduação em Química, 96160-000 Capão do Leão-RS, Brasil.

${ }^{3}$ Aluno de Doutorado - Universidade Federal de Pelotas (UFPel), Laboratório de Metrologia Química, Programa de Pós-Graduação em Química, 96160-000 Capão do Leão-RS, Brasil.

${ }^{4}$ Aluna de Mestrado - Universidade Federal do Rio de Janeiro (UFRJ), LaDANM - Laboratório de Desenvolvimento e Aplicações de Novos Materiais, Instituto de Química, 21941-909 Rio de Janeiro-RJ, Brasil.

${ }^{5}$ Professor Associado - Universidade Federal do Rio de Janeiro (UFRJ), Laboratório de Fotoquímica, Instituto de Química, 21941-909 Rio de Janeiro-RJ, Brasil.

${ }^{6}$ Professora Adjunto - Universidade Federal de Pelotas (UFPel), Laboratório de Metrologia Química, Programa de Pós-Graduação em Química, 96160-000 Capão do Leão-RS, Brasil.

${ }^{7}$ Professor Adjunto - Universidade Federal de Pelotas (UFPel), Laboratório de Metrologia Química, Programa de Pós-Graduação em Química, 96160-000 Capão do Leão-RS, Brasil.

${ }^{8}$ Professor Associado - Universidade Federal do Rio de Janeiro (UFRJ), LaDANM - Laboratório de Desenvolvimento e Aplicações de Novos Materiais, Instituto de Química, 21941-909 Rio de Janeiro-RJ, Brasil. emersonsr@iq.ufrj.br
} 


\section{Introdução}

Com a crescente preocupação com o meio ambiente, as indústrias químicas vêm sofrendo uma constante pressão através da sociedade e das autoridades governamentais para que desenvolvam novas tecnologias, novos materiais e processos químicos mais eficientes do ponto de vista da química verde, que não agridam o meio ambiente (PRADO, 2003). Uma promissora área é a produção de óxidos mistos a base de sílica, os quais se mostram como uma alternativa a esses problemas, uma vez que possuem um grande potencial catalítico, podendo assim ser usado industrialmente (PRADO, 2003; MOROSANOVA, 2012). Esses materiais também podem ser utilizados em reações, por exemplo, de degradação de compostos orgânicos em meio aquoso, sendo esta, uma alternativa para a descontaminação de efluentes em indústrias, como na eliminação de compostos fenólicos (BRITTO; RANGEL, 2008; NDIAYE, et al., 2004).

A vantagem na utilização desses materiais é que são estáveis quimicamente em diferentes meios reacionais e atuam de forma heterogênea, assim possibilitando sua recuperação e reutilização, seguindo um dos princípios da química verde (PRADO, 2003). Além disso, há a possibilidade de inserção de metais em sua estrutura a base de sílica, ou seja, dopagem; onde esses sólidos obtidos, designados de compósitos, normalmente aliam as propriedades químicas dos óxidos metálicos livres (propriedades ácidas de Brønsted ou de Lewis) com as propriedades mecânica e química da sílica gel, o que possibilita atribuir a esses materiais diferentes propriedades como sorção e catálise (CUNDY; COX, 2003; CASTELLANI; GUSHIKEM, 2000).

No entanto, a identificação de metais nesses sólidos inorgânicos, normalmente é feita a partir do uso de técnicas qualitativas ou semiquantitativas, como a espectroscopia fotoeletrônica de raios X (XPS), espectroscopia de dispersão de energia de raios-X (EDS), fluorescência de raios X (XRF) ou espectrometria de retroespalhamento de Rutherford (RBS), aos quais necessitam de grandes quantidades de amostras, que muitas vezes não podem ser obtidos na escala de pesquisa durante o desenvolvimento de novos materiais (MORI; HASEBE; TERANO, 1999; PANDOLFI, et al., 2008; KANDA, et al., 2010; STEDILE; DOS SANTOS, 1998). Com isso, torna-se difícil a caracterização desses materiais, exceto quando o método é realizado em microescala, fazendo uso de poucos miligramas de amostra em análise quantitativas, visando principalmente a etapa de preparo das amostras. Dentre as técnicas analíticas capazes de quantificar metais em silicatos, destaca-se a espectrometria de absorção atômica (AAS), espectrometria de emissão óptica com plasma indutivamente acoplado (ICP-OES) e espectrometria de massa com plasma indutivamente acoplado (ICPMS), as quais se caracterizam por serem sensíveis e reprodutíveis para análises elementares (RESANO, et al., 2012; WANG; TOPHAM; WU, 2011). Sendo que as técnicas de ICP-OES e ICP-MS são as mais empregadas na caracterização de óxidos mistos a base de sílica, com o intuito de quantificar os óxidos metálicos (LIN; BAI, 2016; GOLANDAJ, et al., 2015; ENSAFI, et al., 2013; ARSHADI, et al., 2013). Grande parte dos equipamentos de espectrometria atômica disponíveis nos laboratórios de pesquisa e de rotina na atualidade necessita que a amostra esteja na sua forma líquida para a sua introdução e análise (OLIVEIRA, 2003).

Trabalhos na literatura destacam o uso de análise direta de sólidos em espectrometria de absorção atômica, principalmente devido a equipamentos dedicados comercialmente para este tipo de análise, o que facilita a etapa do preparo das amostras, tornando-a 
mais rápida, econômica e segura, uma vez que assim o risco de contaminação e/ou perda do analito são minimizados (BELARRA, et al., 2002; NOMURA; SILVA; OLIVEIRA, 2008). Entretanto, mesmo sendo mínima, a manipulação das mesmas não é dispensável, uma vez que é necessária uma moagem eficiente para alcançar partículas em tamanhos adequados para as análises. Além disso, alguns pontos negativos podem ser atribuídos a essa técnica, tais como a imprecisão nos resultados devido à falta de homogeneidade, pois essa técnica exige pequenas quantidades de amostras, bem como o surgimento de alguns inconvenientes no momento da calibração do equipamento, visto que pode se tornar complexa dependendo da natureza da amostra, provocando, por exemplo, o efeito de matriz (NOMURA; SILVA; OLIVEIRA, 2008).

A decomposição ácida caracteriza-se como um método adequado para o tratamento de amostras de sílicas ou óxidos mistos a base de sílica gel, que comumente é executada com a adição de ácido fluorídrico (HF) para essas amostras (XU, et al., 2005; WANG; TOPHAM; WU, 2011). Contudo, uma atenção maior deve ser voltada para o ácido fluorídrico quando utilizado em concentrações elevadas, pois pode levar a corrosão de peças instrumentais, bem como colocar em perigo o operador (WANG; TOPHAM; WU, 2011).

O presente trabalho tem por objetivo avaliar a metodologia do preparo das amostras em microescala, buscando aliar a simplicidade e utilizar a mínima quantidade de amostra possível, na ordem de miligramas, compatível com a escala de pesquisa laboratorial, além de fazer uso de uma quantidade reduzida de reagentes, o que está diretamente relacionado com a quantidade de resíduos gerados, contribuindo de forma efetiva para a química verde. Para tais amostras, foram determinadas as concentrações totais de Mn e Zn utilizando a técnica de espectrometria de absorção atômica em chama (FAAS).

\section{Experimental}

\section{Equipamentos e parâmetros instrumentais}

Todas as medidas de quantificação de Mn e Zn foram realizadas utilizando um espectrômetro de absorção atômica modelo AA-6300 (Shimadzu, Japão) equipado com um amostrador automático modelo ASC-6100, uma lâmpada de arco de deutério como corretor de fundo e lâmpadas de cátodo oco para Mn e Zn (Hamamatsu photonics K.K, Japão). Para este estudo, foi adotado um acessório disponibilizado neste modelo de espectrômetro, o qual permite a injeção de pequenos volumes $(5-200 \mu \mathrm{L})$ de solução da amostra na chama, o que permite registrar o sinal analítico através da integração em área de um sinal transiente. $\mathrm{O}$ espectrômetro foi operado conforme as seguintes condições: comprimento de onda de 279,48 e 213,86 nm e corrente da lâmpada: $10 \mathrm{~mA}$ para Mn e Zn, respectivamente. A chama utilizada no presente estudo por FAAS foi ar/acetileno.

Foram utilizados os equipamentos de Fluorescência de Raios X por dispersão de energia para caracterização dos óxidos mistos inorgânicos, o primeiro da marca Shimadzu, modelo: EDX 800 HS, e o segundo da marca Rigaku, modelo: RIX 3100 com tubo de ródio de $4 \mathrm{~kW}$.

Para a microdigestão ácida das amostras em micro frascos de polipropileno (PP) foi utilizado um forno de micro-ondas doméstico (Panasonic, Manaus, AM, Brasil, Modelo NN-ST571WRU de frequência de $2450 \mathrm{MHz}$ ).

\section{Reagentes e soluções}

Todos os reagentes utilizados foram de grau analítico. A água deionizada para o preparo das amostras e das soluções foi obtida por um sistema de purificação de água Direct-Q 3 com resistividade de 
18,3 M $\Omega \mathrm{cm}$ (Millipore Corporation, Bedford, MA, U.S.A.). Ácido nítrico $65 \%$ (m/m) (Synth, Brasil) foi purificado por destilação em um sistema de subebulição de quartzo MA-075 (Marconi, Piracicaba, SP, Brasil). Além desse, também foi utilizado para o processo de decomposição das amostras o ácido fluorídrico P.A. (Vetec, Brasil) e peróxido de hidrogênio (Vetec, Brasil). Soluções de $\mathrm{Mn}$ e $\mathrm{Zn}$ foram diariamente preparadas por diluição de solução estoque padrão contendo $1000 \mathrm{mg} \mathrm{L}^{-1}$ (Merck, Darmstadt, Alemanha) de cada elemento.

Vidrarias e recipientes de plásticos foram imersos em um banho de $\mathrm{HNO}_{3} 10 \%$ (v/v), por no mínimo 48 horas e, posteriormente, lavados com água deionizada e secos antes do uso.

\section{Sínteses dos óxidos mistos obtidos pelo processo sol-gel}

Todos os óxidos mistos utilizados no trabalho foram obtidos pelo processo sol-gel, seguindo procedimentos anteriormente descritos e desenvolvidos pelo Grupo LaDANM - Laboratório de Desenvolvimento e Aplicações de Novos Materiais (DINIZ, et al., 2014; GARRIDO, et al., 2013).

Os óxidos mistos $\mathrm{SiO}_{2} / \mathrm{Nb}_{2} \mathrm{O}_{5} / \mathrm{ZnO}$ em duas diferentes proporções em massa, denominados de SiNbZn-A e SiNbZn-B, foram sintetizados seguindo o procedimento descrito por Diniz e colaboradores (DINIZ, et al., 2014).

Os demais óxidos mistos contendo $\mathrm{MnO}_{\mathrm{x}}$ foram sintetizados seguindo o procedimento descrito por Garrido e colaboradores (GARRIDO, et al., 2013); sendo denominados de SiAlMn (referente ao óxido misto $\mathrm{SiO}_{2} /$ $\mathrm{Al}_{2} \mathrm{O}_{3} / \mathrm{MnO}_{\mathrm{x}}$ ); SiMnC-A e SiMnC-B (referente ao óxido misto $\mathrm{SiO}_{2} / \mathrm{MnO}_{\mathrm{x}} /$ Grafite sintetizado em duas diferentes proporções em massa) e SiMn-A, SiMn-B e SiMn-C (referente $\mathrm{SiO}_{2} / \mathrm{MnO}_{\mathrm{x}}$ sintetizados a partir de dois diferentes precursores de $\mathrm{Mn}(\mathrm{II})$ : $\left(\mathrm{CH}_{3} \mathrm{CO}_{2}\right)_{2} \mathrm{Mn} \cdot 4 \mathrm{H}_{2} \mathrm{O}$, SiMn-A ou Mn( $\left(\mathrm{NO}_{3}\right)_{2} \cdot 6 \mathrm{H}_{2} \mathrm{O}$, SiMn-B e SiMn-C).

\section{Preparo das Amostras}

As amostras de óxido misto foram decompostas individualmente e diretamente em frascos de polipropileno (PP) de 2,0 mL. Foram pesadas aproximadamente $10 \mathrm{mg}$ das amostras e adicionados $50 \mu \mathrm{L}$ de $\mathrm{HF}, 50 \mu \mathrm{L}$ de $\mathrm{HNO}_{3}$ $65 \%(\mathrm{~m} / \mathrm{m})$ e $25 \mu \mathrm{L}$ de $\mathrm{H}_{2} \mathrm{O}_{2}$, as misturas foram levadas a um forno de micro-ondas e posicionadas individualmente no centro do prato no interior do forno, conforme sugerido por Rosini et al. (ROSINI; NASCENTES; NÓBREGA, 2012), os quais evidenciaram que este é o local de melhor distribuição da radiação micro-ondas. Para tal processo, as amostras permaneceram por 120 segundos na potência de 700 Watts. Após a mineralização das amostras, a solução resultante foi então avolumada a 2,0 mL com água deionizada. A adição do $\mathrm{H}_{2} \mathrm{O}_{2}$ foi utilizada no intuito de regenerar $\mathrm{OHNO}_{3}$ a partir de $\mathrm{NO}_{2}$ formado no meio reacional, auxiliando também na redução da pressão do frasco fechado. Não sabemos se isso realmente possa ter ocorrido neste sistema, mas como a decomposição do $\mathrm{H}_{2} \mathrm{O}_{2}$ tem como produto final somente água e a solução resultante da decomposição seria avolumada com água deionizada, optamos por utilizar com base no trabalho realizado por Miranda e colaboradores (MIRANDA; PEREIRA-FILHO; NETO, 2014).

Para checagem da exatidão, realizou o procedimento de adição e recuperação, onde a massa dos analitos e amostra foram consideradas para os valores de adição, sendo que a adição foi realizada logo após a pesagem da amostra, antes de todas as outras etapas. Para os cálculos, as curvas de calibração eram em

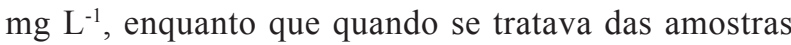
em $\mathrm{g} \mathrm{Kg}^{-1}$, o ajuste de unidade era feito por cálculos considerando as diluições e massas dos analitos e ou 
das amostras.

As amostras estudadas não contêm matéria orgânica, o que diminui o risco de explosão, uma vez que não há a geração de $\mathrm{CO}_{2}$. Vale ressaltar também, que o procedimento em microescala torna esse risco praticamente inexistente, ao contrário das unidades comerciais de preparo de amostra, que geralmente empregam massas bem superiores, necessitando de um rigoroso controle de temperatura e pressão e válvulas de segurança. Além disso, após a etapa de aquecimento, os minis frascos foram mantidos em repouso por pelo menos $30 \mathrm{~min}$, para que os mesmos pudessem ser arrefecidos até temperatura ambiente, antes da sua abertura.

Após o preparo das amostras, as soluções foram encaminhadas para análise instrumental e as concentrações dos analitos foram determinadas a partir da construção de curvas de calibração no mesmo meio de preparo das amostras. O volume de amostra e soluções de calibração utilizadas para todas as leituras foi de $200 \mu \mathrm{L}$.

\section{Resultados e discussões}

Caracterização dos óxidos mistos obtidos pelo processo sol-gel por XRF

A análise química do percentual em massa do $\mathrm{ZnO}$ nos materiais SiNbZn-A e SiNbZn-B foi realizada com o auxílio de um equipamento de fluorescência de raios X da marca Shimadzu. As amostras (cerca de 100 mg do óxido misto) foram prensadas usando ácido bórico como substrato sob pressão de 20 toneladas. As análises foram realizadas em colaboração com o Instituto de Engenharia Nuclear (IEN).
Tabela 1: Resultados obtidos por XRF do percentual em massa $(\mathrm{X} \pm \mathrm{SD})$ dos óxidos de $\mathrm{ZnO}$ e $\mathrm{MnO}_{\mathrm{x}}$ nos materiais obtidos pelo processo sol-gel comparado com os valores teóricos esperados na síntese.

\begin{tabular}{lcccc}
\hline Material & $\begin{array}{c}\mathrm{MnO}_{\mathrm{x}}- \\
\text { teórica } \\
\left(\mathrm{mg} \mathrm{g}^{-1}\right)\end{array}$ & $\begin{array}{c}\mathrm{ZnO}- \\
\text { teórica } \\
\left(\mathrm{mg} \mathrm{g}^{-1}\right)\end{array}$ & $\begin{array}{c}\mathrm{MnO}_{\mathrm{x}}-\mathrm{XRF} \\
\left(\mathrm{mg} \mathrm{g}^{-1}\right)\end{array}$ & $\begin{array}{c}\mathrm{ZnO}-\mathrm{XRF} \\
\left(\mathrm{mg} \mathrm{g}^{-1}\right)\end{array}$ \\
\hline SiNbZn-A & 0 & 100,0 & nd & $101,1 \pm 0,01$ \\
SiNbZn-B & 0 & 200,0 & nd & $193,9 \pm 0,02$ \\
SiAlMn & 200,0 & 0 & nd & nd \\
SiMnC-A & 200,0 & 0 & nd & nd \\
SiMnC-B & 200,0 & 0 & nd & nd \\
SiMn-A & 200,0 & 0 & $102,1 \pm 0,01$ & nd \\
SiMn-B & 300,0 & 0 & $203,4 \pm 0,02$ & nd \\
SiMn-C & 300,0 & 0 & $377,1 \pm 0,04$ & nd \\
\hline
\end{tabular}

nd: Valores não determinados por fluorescência de raios X. Fonte: Autor

A análise química do percentual em massa dos $\mathrm{MnO}_{\mathrm{x}}$ nos materiais SiMn-A, SiMn-B e SiMn-C foram também realizadas com o auxílio de um equipamento de fluorescência de raios X. Nestas análises, as amostras foram obtidas por prensagem dos materiais sob pressão de 20 toneladas. As pastilhas obtidas foram analisadas em um espectrômetro de fluorescência de raios $\mathrm{X}$, da marca Rigaku. Na obtenção da análise química semiquantitativa, o espectro gerado a partir da amostra foi avaliado pelo software Spectraplus v.1.6 no modo standardlessmethod, ou seja, sem curva de calibração específica.

Os resultados obtidos após análise dos óxidos mistos por fluorescência de raios $\mathrm{X}$ estão apresentados na Tabela 1. Como pode ser visto, os valores obtidos por XRF para o $\mathrm{ZnO}$ nos materiais SiNbZn-A e SiNbZn-B coincidem com os valores teóricos, mostrando que a metodologia de síntese é eficiente para a preparação desses materiais. No entanto, para os materiais SiMn-A, SiMn-B e SiMn-C, os valores obtidos por XRF para o $\mathrm{MnO}_{\mathrm{x}}$ não coincidem com os valores teóricos, indicando que a metodologia 
de síntese é menos efetiva para a preparação desses materiais; sugerindo que a hidrólise dos precursores de $\mathrm{SiO}_{2}$ e $\mathrm{MnO}_{x}$ não ocorre totalmente.

Por outro lado, apesar dos resultados obtidos por XRF serem próximos para os materiais SiNbZn-A e SiNbZn-B e discordantes para os materiais SiMn-A, SiMn-B e SiMn-C; estes resultados podem não ser satisfatórios, pois diversos fatores afetam a análise quantitativa, dentre os quais podemos destacar principalmente a dificuldade de calibração, onde muitas vezes são utilizados óxidos metálicos ou o metal puro, e que não consegue reproduzir as diferentes matrizes das amostras durante o processo de calibração. Tal efeito pode ocorrer simplesmente pelas diferentes densidades entre as amostras, afetando a incidência de radiação e atuando cada vez mais próximo a superfície. Além disso, dependendo da síntese, o metal pode estar ocluído internamente nas partículas sólidas, dificultando a sua detecção, enquanto em outras, pode estar na superfície e de diferentes formas, afetando sem dúvida o resultado das análises. Conforme relata Lima e colaboradores (LIMA, et al., 2013) valores obtidos por estudos experimentais por EPR (espectroscopia de ressonância paramagnética eletrônica), os materiais contendo óxido de manganês, pode se apresentar como uma mistura de $\mathrm{Mn}(\mathrm{II})$ e $\mathrm{Mn}(\mathrm{III})$, possuindo clusters de $\mathrm{Mn}_{3} \mathrm{O}_{4}$, que é a mistura desses óxidos, por isso é melhor representado como $\mathrm{MnO}_{\mathrm{x}}$.

\section{Resultados das análises obtidos por FAAS}

Os parâmetros de mérito obtidos por F AAS estão apresentados na Tabela 2, sendo que se obtiveram as concentrações características de 0,024 e $0,031 \mathrm{mg} \mathrm{L}^{-1}$ para o Zn e Mn, respectivamente e a frequência analítica do método proposto é de 40 determinações por hora. Para o cálculo do limite de detecção foi utilizada a fórmula:

$$
L D=\frac{3 \cdot S D}{s}
$$

Onde:

$\mathrm{SD}$, desvio padrão da leitura de dez sinais do branco das amostras; s, sensibilidade da curva de calibração.

Tabela 2: Parâmetros de mérito para a determinação de $\mathrm{Mn}$ e $\mathrm{Zn}$ em amostras de sílica por FAAS.

\begin{tabular}{ccccc}
\hline Analito & $\begin{array}{c}\text { Faixa de } \\
\text { Calibração } \\
\left(\mathrm{mg} \mathrm{L}^{-1}\right)\end{array}$ & $\begin{array}{c}\text { Sensibilidade } \\
\left(\mathrm{L} \mathrm{mg}^{-1}\right)\end{array}$ & $\mathrm{R}$ & $\begin{array}{c}\text { LD } \\
(\mathrm{mg} \\
\left.\mathrm{g}^{-1}\right)\end{array}$ \\
\hline $\mathrm{Mn}$ & $1-5$ & 0,14 & 0,9957 & 0,098 \\
$\mathrm{Zn}$ & $0,5-1$ & 0,18 & 0,9976 & 0,006 \\
\hline
\end{tabular}

$\mathrm{R}$ : Coeficiente de correlação linear. $\mathrm{LD}_{(\mathrm{m})}=\mathrm{LD}$ do método. Fonte: Autor

Diferentes amostras decompostas em microescala contendo $\mathrm{Mn}$ ou $\mathrm{Zn}$ foram analisadas através de microinjeção por FAAS, e os resultados para a concentração desses analitos nas amostras investigadas, com e sem adição do analito, estão apresentados na Tabela 3.

A metodologia estudada foi avaliada com teste de adição, sendo que a quantidade de 0,6 e $0,1 \mathrm{mg} \mathrm{g}^{-1}$ para $\mathrm{Mn}$ e $\mathrm{Zn}$ foram adicionadas em todas as amostras e os resultados de recuperação foram satisfatórios, uma vez que foi possível alcançar recuperações na faixa de 93,5 a 100,7 \%, conforme os resultados apresentados na Tabela 3. Além disso, é possível notar uma boa precisão entre os resultados obtidos de acordo com a Tabela 3, pois a média dos RSD's obtidos foi em torno de 5,0 e $8,7 \%$ para $\mathrm{Zn}$ e Mn, respectivamente, mesmo utilizando uma metodologia que empregou o preparo de amostra em microescala, aliada a micro injeção no FAAS.

Dessa forma, o procedimento de micro-injeção da amostra necessita de pequenos volumes para análise e resulta em procedimento que geram uma menor quantidade de resíduos e se apresenta como uma excelente alternativa 
aos princípios da química verde, dispensando o uso de procedimentos convencionais

Tabela 3: Resultados para Mn e $\mathrm{Zn}$ nas amostras de sílica por FAAS, $n=3$.

\begin{tabular}{|c|c|c|c|c|}
\hline Amostra & $\begin{array}{l}\text { Adição } \\
\left(\mathrm{mg} \mathrm{g}^{-1}\right)\end{array}$ & $\begin{array}{c}\text { Concentração, } \mathrm{x} \\
\pm \mathrm{SD} \\
\left(\mathrm{mg} \mathrm{g}^{-1}\right)\end{array}$ & $\begin{array}{c}\text { RSD } \\
(\%)\end{array}$ & $\begin{array}{c}\text { Recuperação } \\
(\%)\end{array}$ \\
\hline \multicolumn{5}{|c|}{ Determinação de $\mathrm{Zn}$} \\
\hline \multirow{2}{*}{ SiNbZn-A } & 0 & $0,196 \pm 0,013$ & 6,6 & \\
\hline & 0,100 & $0,293 \pm 0,029$ & 10,0 & 96,3 \\
\hline \multirow{2}{*}{ SiNbZn-B } & 0 & $33,8 \pm 1,2$ & 3,6 & \\
\hline & 0,100 & $33,9 \pm 2,7$ & 8,0 & 93,5 \\
\hline \multicolumn{5}{|c|}{ Determinação de Mn } \\
\hline \multirow{2}{*}{ SiAlMn } & 0 & $54,3 \pm 4,2$ & 7,7 & \\
\hline & 0,600 & $54,9 \pm 0,9$ & 1,6 & 94,7 \\
\hline \multirow{2}{*}{ SiMnC-A } & 0 & $10,8 \pm 1,1$ & 10,2 & \\
\hline & 0,600 & $11,4 \pm 0,8$ & 7,0 & 95,6 \\
\hline \multirow{2}{*}{ SiMnC-B } & 0 & $7,99 \pm 0,24$ & 3,0 & \\
\hline & 0,600 & $8,59 \pm 0,35$ & 4,1 & 99,9 \\
\hline \multirow{2}{*}{ SiMn-A } & 0 & $34,8 \pm 2,9$ & 8,3 & \\
\hline & 0,600 & $35,3 \pm 1,8$ & 5,1 & 97,2 \\
\hline \multirow{2}{*}{ SiMn-B } & 0 & $94,6 \pm 8,6$ & 9,1 & \\
\hline & 0,600 & $95,1 \pm 9,8$ & 10,3 & 94,2 \\
\hline \multirow{2}{*}{ SiMn-C } & 0 & $97,9 \pm 5,6$ & 5,7 & \\
\hline & 0,600 & $98,5 \pm 7,5$ & 7,6 & 100,7 \\
\hline
\end{tabular}

Média \pm desvio padrão; RSD (Desvio padrão relativo).

Fonte: Autor

Dessa forma, vale ressaltar que as técnicas de análise qualitativa podem indicar ou não a presença do metal no material sintetizado, além de não serem técnicas destrutivas e o material pode ser reaproveitado. Por outro lado, quando necessário uma análise quantitativa, na maioria das vezes é necessário transformar a amostras para uma forma viável para ser introduzida no equipamento de medida, levando a sua destruição, dessa forma o uso de métodos em microescala, possibilita que está quantificação utilize a mínima quantidade do material, principalmente durante o desenvolvimento de novos materiais em pequena escala de produção durante as pesquisa, possibilitando que a alíquota utilizada não consuma todo o material produzido, o que poderia inviabilizar os estudos de caracterização por outras técnicas de análise, além de testes das possíveis aplicações.

\section{Conclusão}

Ametodologiadesenvolvidaparao preparodeamostras a base de sílica em microescala, com decomposição assistida por micro-ondas, mostrou ser eficiente, simples, rápida e reprodutível para a quantificação dos elementos Mn e Zn nesses materiais inorgânicos analisados por FAAS. A quantidade de amostra comumente disponível durante o desenvolvimento destes materiais, não permitiriam o uso de procedimento convencional de preparo de amostra, que geralmente utilizam massas com pelo menos 10 vezes superiores a utilizada neste trabalho. Certamente a metodologia estudada poderá ser estendida para a determinação de outros elementos que possam ser inseridos nesse suporte.

Portanto, mostra-se um procedimento adequado para o objetivo do trabalho, podendo auxiliar de forma efetiva na caracterização destes novos materiais compatível com a escala de pesquisa, o qual muitas vezes dispõe de pouca amostra para sua caracterização e controle de qualidade. Além disso, foi requerido um pequeno volume da solução de amostra para as análises por FAAS, evitando desperdícios, tanto de reagentes como também de amostras, e geração de grandes volumes de resíduos. Ampliando os estudos, essa metodologia também pode ser empregada para quantificação de metais tóxicos adsorvidos por zeólitas em águas residuais ou contaminada, permitindo assim o controle ambiental.

\section{Agradecimentos}

Os autores são gratos ao Conselho Nacional de Desenvolvimento Científico e Tecnológico (CNPq) e a Coordenação de Aperfeiçoamento de Pessoal de Nível Superior (CAPES) por bolsas concedidas e ao CNPq pelo suporte financeiro (Processo ${ }^{\circ}$ 447373/2014-5).

\section{Referências}

ARSHADI, M.; VAHID, F. S.; SALVACION, J. W. L.; SOLEYMANZADEH, M. A practical organomettalic decorated nano-size $\mathrm{SiO}_{2}-\mathrm{Al}_{2} \mathrm{O}_{3}$ mixed-oxides for methyl 
orange removal from aqueous solution. Applied Surface Science, v. 280, p. 726-736, 2013.

BELARRA, M. A.; RESANO, M.; VANHAECKE, F.; MOENS, L. Direct solid sampling with electrothermal vaporization/atomization: what for and how?. Trac-Trend in Analytical Chemistry, v. 21, p. 828-839, 2002.

BRITTO, J. M.; RANGEL, M. C. Advanced oxidation process of phenolic compounds in industrial wasterwater. Química Nova, v. 31, p. 114-122, 2008.

CASTELLANI, A. M.; GUSHIKEM, Y. Electrochemical properties of a porphyrin-cobalt (II) adsorbed on silicatitania-phosphate composite surface prepared by the sol-gel method. Journal of Colloid and Interface Science, v. 230, p. 195-199, 2000.

CUNDY, C. S.; COX, P. A. The hydrothermal synthesis of zeolites: History and development from the earliest days to the present time. Chemical Reviews, v. 103, p. 663-701, 2003.

DINIZ, K. M.; GORLA, F. A.; RIBEIRO, E. S.; do NASCIMENTO, M. B. O.; CORREA, R. J.; TARLEY, C. R. T.; SEGATELLI, M. G. Preparation of $\mathrm{SiO}_{2} / \mathrm{Nb}_{2} \mathrm{O}_{5} /$ $\mathrm{ZnO}$ mixed oxide by sol-gel method and its application for adsorption studies and on-line preconcentration of cobalt ions from aqueous médium. Chemical Engineering Journal, v. 239, p. 233-241, 2014.

ENSAFI, A. A.; GHIACI, M.; ARSHADI, M.; KARIMI-MALEH, H. Synthesis and characterization of ferrocenecarboxaldehyde immobilized on modified $\mathrm{SiO}_{2}-\mathrm{Al}_{2} \mathrm{O}_{3}$ in nanoscale, application for determination of penicillamine. Journal of Nanoparticle Research, v. 15, artigo 1610, 2013.

GARRIDO, F. M. S.; MEDEIROS, R. F.; NOGUEIRA, N. O. B.; PERES, R. C. D.; RIBEIRO, E. S.; MEDEIROS,
M.E. Síntese de óxidos mistos $\mathrm{SiO}_{2} / \mathrm{Mn}_{\mathrm{x}} \mathrm{Oy}$ para aplicação na reação de redução de $\mathrm{O}_{2}$. Revista Matéria, v. 18, p. 1294-1305, 2013.

GOLANDAJ, A. J.; MAHOMED, A. S.; SINGL, S.; FRIEDRICH, H. B. Effect of different weight loadings of $\mathrm{MoO}_{\mathrm{x}} / \mathrm{SBA}-15$ on the oxidative dehydrogenation of n-octane. Journal of Porous Materials, v. 22, p. 787-796, 2015.

KANDA, Y.; TEMMA, C.; NAKATA, K.; KOBAYASHI, T.; SUGIOKA, M.; UEMICHI, Y. Preparation and performance of noble metal phosphides supported on silica as new hydrodesulfurization catalysts. Applied Catalysis A-General, v. 386, p. 171-178, 2010.

LIMA, G. F.; FERREIRA, V. S.; GODOY, N. V.; MEDEIROS, R. F.; GARRIDO, F. M. S.; RIBEIRO, E. S.; NAKAGAKI, S.; SEGATELLI, M. G.; BEZERRA, M. A.; TARLEY, C. R. T. Study of silica-manganese oxide hybrid material as a new solid phase for on-line continuous flow enrichment of $\mathrm{Cd}(\mathrm{II})$ ions coupled to flame atomic absorption spectrometry. Microchemical Journal, v. 109, p. 98-105, 2013.

LIN, L. Y.; BAI, H. L. Salt-induced formation of hollow and mesoporous $\mathrm{CoO}_{x} / \mathrm{SiO}_{2}$ spheres and their catalytic behavior in toluene oxidation. RSC Advances, v. 6, p. 24304-24313, 2016.

MIRANDA, K.; PEREIRA-FILHO, E. R.; GOMES NETO, J. A. A new closed-vessel conductively heated digestion system: fostering plant analysis by inductively coupled plasma optical emission spectroscopy. Journal of Analytical Atomic Spectrometry, v. 29, p. 825-831, 2014.

MORI, H.; HASEBE, K.; TERANO, M. XPS study of the interaction of titanium species with internal electron donors on $\mathrm{MgCl} 2$-supported Ziegler catalysts. Journal of Molecular Catalysis A-Chemical, v. 140, p. 165-172, 
1999.

MOROSANOVA, E. I. Silica and silica-titania sol-gel materials: Synthesis and analytical application. Talanta, v. 102, p. 114-122, 2012.

NDIAYE, P. L.; MOULIN, P.; DOMINGUEZ, L.; MILLET, J. C.; CHARBIT, F. Treatment of silica effluents: ultrafiltration or coagulation-decantation. Journal of Hazardous Materials, v. 116, p. 75-81, 2004.

NOMURA, C. S.; SILVA, C. S.; OLIVEIRA, P. V. Solid sampling graphite furnace atomic absorption spectrometry: A review. Química Nova, v. 31, p. 104113, 2008.

OLIVEIRA, E. Sample preparation for atomic spectroscopy: Evolution and future trends. Journal of the Brazilian Chemical Society, v. 14, p. 174-182, 2003.

PANDOLFI, L.; CAFARELLI, P.; KACIULIS, S.; TOMLINSON, A. A. G. XPS analysis of several zeolitic and clay-based nanoporous materials for C4 hydrocarbon conversions. Microporous and Mesoporous Materials, v. 110, p. 64-71, 2008.

PRADO, A. G. S. Green chemistry, the chemical challenges of the new millenium. Química Nova, v. 26, p. 738-744, 2003.

RESANO, M.; MOZAS, E.; CRESPO, C.; PEREZ, J.; GARCIA-RUIZ, E.; BELARRA, M. A. Direct analysis of silica by means of solid sampling graphite furnace atomic absorption spectrometry. Spectrochimica Acta Part B-Atomic Spectroscopy, v. 71-72, p. 24-30, 2012.

ROSINI, F.; NASCENTES, C. C.; NOBREGA, J. A. Microwave-assisted experiments for undergraduate courses. Química Nova, v. 27, p. 1012-1015, 2004.

STEDILE, F. C.; SANTOS, J. H. Z. Analysis and characterization of real catalysts using ion beam analysis. Nuclear Instruments \& Methods in Physics Research Section B-Beam Interactions whith Materials and Atoms, v. 136, p. 1259-1266, 1998.

WANG, J.; TOPHAM, N.; WU, C. Y. Determination of silica coating efficiency on metal particles using multiple digestion methods. Talanta, v. 85, p. 26552661,2011

XU, Y. H.; IWASHITA, A; NAKAJIMA, T.; YAMASHITA, H.; TAKANASHI, H.; OHKI, A. Effect of $\mathrm{HF}$ addition on the microwave-assisted acid-digestion for the determination of metals in coal by inductively coupled plasma-atomic emission spectrometry. Talanta, v. 66, p. $58-64,2005$.
Recebido em 18 Maio, 2016 - Received on May 18, 2016 Aceito em 13 Outubro, 2016-Accepted on October 13, 2016 
